\title{
Parity-Violating Møller Scattering at Next-to-Next-to-Leading Order: Closed Fermion Loops
}

\author{
Yong Du๑,$^{1, *}$ Ayres Freitas, ${ }^{2, \dagger}$ Hiren H. Patel, ${ }^{3, *}$ and Michael J. Ramsey-Musolf ${ }^{4,1,5,8}$ \\ ${ }^{1}$ Amherst Center for Fundamental Interactions, Physics Department, University of Massachusetts Amherst, \\ Amherst, Massachusetts 01003 USA \\ ${ }^{2}$ Pittsburgh Particle Physics Astrophysics and Cosmology Center (PITT-PACC), Department of Physics and Astronomy, \\ University of Pittsburgh, Pittsburgh, Pennsylvania 15260, USA \\ ${ }^{3}$ Department of Physics and Santa Cruz Institute for Particle Physics, University of California, Santa Cruz, California 95064, USA \\ ${ }^{4}$ Tsung-Dao Lee Institute and School of Physics and Astronomy, Shanghai Jiao Tong University, Shanghai 200240, China \\ ${ }^{5}$ Kellogg Radiation Laboratory, California Institute of Technology, Pasadena, California 91125 USA
}

(Received 17 January 2020; revised 22 July 2020; accepted 23 February 2021; published 29 March 2021)

A complete, gauge-invariant computation of two-loop virtual corrections involving closed fermion loops to the polarized Møller scattering asymmetry is presented. The set of contributions involving two closed fermion loops and the set involving one closed fermion loop are numerically similar in magnitude to the one-loop bosonic corrections and yield an overall correction of $1.3 \%$ relative to the tree level asymmetry. We estimate sizes of the remaining two-loop contributions and discuss implications for the upcoming MOLLER (Measurement of a Lepton-Lepton Electroweak Reaction) experiment.

DOI: 10.1103/PhysRevLett.126.131801

Introduction.-Precision measurements of electroweak processes have played a vital role in the development and testing of the standard model (SM) of particle physics. With the discovery of the Higgs boson at the CERN Large Hadron Collider, the focus of precision tests now falls squarely on the search for signs of physics beyond the SM (BSM). While a variety of open questions clearly point to the existence of BSM physics, it remains to be determined at what mass scale this physics lives and how it interacts with the known elementary particles of the SM.

A powerful probe in this context is parity-violating electron scattering (PVES). The relevant observable in PVES experiments is the asymmetry $A_{L R}$ in the crosssection when otherwise identical beams of longitudinally polarized electrons of left $(L)$ and right $(R)$ helicities scatter from a fixed target:

$$
A_{L R}=\frac{d \sigma_{L}-d \sigma_{R}}{d \sigma_{L}+d \sigma_{R}}
$$

Historically, the measurement $A_{L R}$ in deep-inelastic electron-deuteron scattering singled out the GlashowWeinberg-Salam theory [1-3] of the electroweak interaction from other alternatives and provided the first measurement of the all-important weak mixing angle,

Published by the American Physical Society under the terms of the Creative Commons Attribution 4.0 International license. Further distribution of this work must maintain attribution to the author(s) and the published article's title, journal citation, and DOI. Funded by SCOAP ${ }^{3}$.
$\theta_{W}$. Improved results were later obtained by a variety of PVES measurements at low energies, along with observations of parity violation in atomic Cesium and neutrinonucleus deep-inelastic scattering. Parity-violating (PV) Møller scattering provides one of the theoretically cleanest such tests. The first measurement of this asymmetry was made by the E158 Collaboration at SLAC in the mid-2000s [4], yielding a confirmation of the predicted running of $\sin ^{2} \theta_{W}$ with $6 \sigma$ significance.

A new, more precise measurement of the PV Møller asymmetry-dubbed the MOLLER (Measurement of a Lepton-Lepton Electroweak Reaction) experiment and approved to run at the Jefferson Lab [5,6] - aims to determine $A_{L R}$ with a $2.4 \%$ uncertainty. Assuming only SM contributions, the MOLLER experiment will yield a value of $\sin ^{2} \theta_{W}$ with an uncertainty comparable to the earlier determinations in high energy $e^{+} e^{-}$annihilation. Within the SM, this measurement can be interpreted as a precision test of the scale dependence of $\sin ^{2} \theta_{W}$ [7-9]. Its value at $\mu=m_{Z}$ can be obtained either from fits to high energy electroweak precision observables, while PVES experiments yield $\sin ^{2} \theta_{W}$ at a low scale $\mu \ll m_{Z}$.

More significantly, the MOLLER experiment will provide a new probe for BSM physics that could reside at high or low mass scales. Examples include 1-10 TeV doubly charged scalar bosons that are implied by left-right symmetric models for the nonvanishing neutrino masses [10] and a light "dark" $Z$ boson that, under certain conditions, may also account for the observed deviation of the muon anomalous magnetic moment from SM predictions [11-13]. In both examples, the PV Møller asymmetry 
provides a complementary probe to other tests at low and high energies.

The unique potential of the PV Møller scattering follows from two features: the purely leptonic character of the process and a fortuitous suppression of the leading-order asymmetry by $1-4 \sin ^{2} \theta_{W}\left(\sin ^{2} \theta_{W}\right.$ is numerically close to 1/4). Specifically, the theoretical prediction for the PV Møller asymmetry can be written as [14]

$A_{L R}=\frac{G_{\mu} Q^{2}}{\sqrt{2} \pi \alpha} \frac{1-y}{1+y^{4}+(1-y)^{4}}\left(1-4 \sin ^{2} \theta_{W}+\Delta Q_{W}^{e}\right)$,

where $y=Q^{2} / s$ and $\Delta Q_{W}^{e}$ accounts for radiative corrections.

Some terms (SM and possibly BSM) entering through $\Delta Q_{W}^{e}$ do not carry the factor $1-4 \sin ^{2} \theta_{W}$ and thus their relative impact is enhanced. Importantly, the next-leadingorder (NLO) electroweak corrections, whose relative impact should be nominally $\mathcal{O}(\alpha) \sim 0.01$, are roughly $40 \%$ in magnitude [15]. These corrections are dominated by contributions from closed fermion loops that enter the running of $\sin ^{2} \theta_{W}$. The $W W$ and $\gamma Z$ boxes also produce sizeable corrections. Given this enhanced NLO sensitivity, it is important to determine the magnitude of next-to-nextto-leading-order (NNLO) SM corrections if one wishes to interpret correctly a $2.4 \%$ measurement of $A_{L R}$ in terms of BSM physics. Partial results at the NNLO level have been presented in Refs. [16-19]. Furthermore, second-order QED effects have been studied in the context of electron-proton scattering [20], which shares many features with electron-electron scattering.

In what follows, we report on a computation of all NNLO contributions involving closed fermion loops. This subset of the complete NNLO electroweak corrections is gauge-invariant and thus constitutes a well-defined contribution to the asymmetry. Since closed fermion loops dominate the NLO corrections and since they entail a sum over all colors and flavors of SM fermions, we expect them to generate the leading effect at NNLO. We find a resulting $1.3 \%$ correction to the leading-order asymmetry, again significantly larger than one might expect based on $\alpha / 4 \pi$ counting. As we discuss below, we expect the contributions from the remaining NNLO corrections to be smaller in magnitude. We thus anticipate that the overall uncertainty in the SM prediction for $A_{L R}$ lies below the planned experimental uncertainty.

Method.-We calculate the left-right asymmetry by expanding Eq. (1) up to two-loop order. Nonvanishing contributions to $A_{L R}$ arise from the interference of a purely electromagnetic amplitude with the PV component of the weak neutral current amplitude arising from the $Z$ exchange, with the electromagnetic contributions dominating the denominator in Eq. (1). For these building blocks, the NNLO corrections to $d \sigma$ stem from two-loop matrix elements

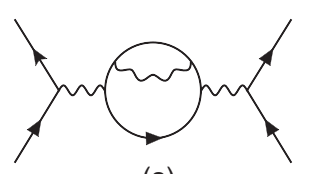

(a)

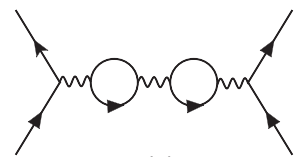

(c)

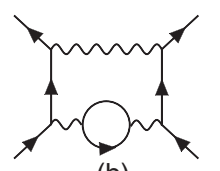

(b)

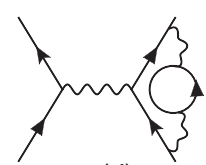

(d)

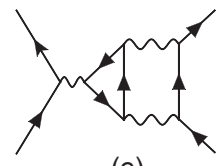

(e)
FIG. 1. Examples of two-loop Feynman diagrams with at least one closed fermion loop for (a) two-loop self-energies, (b) two-loop box contributions, (c) reducible contributions, and $(\mathrm{d}, \mathrm{e})$ two-loop vertex corrections.

contracted with Born amplitudes, as well as the interference of two one-loop matrix elements. The two-loop matrix elements receive contributions from genuine two-loop selfenergy, vertex, and box diagrams and from one-particle reducible two-loop diagrams (see Fig. 1 for examples).

When counting the numbers of fermion loops, we do so at the level of the final observable $A_{L R}$. This means that we include contributions from two-loop diagrams with at least one closed fermion loop, as well as products of a one-loop diagram with a fermion loop with another one-loop diagram. However, for consistency we exclude products of one-loop diagrams without closed fermion loops that could arise from interference terms obtained by expanding the denominator of Eq. (1) to two-loop order.

Logarithmically enhanced contributions from virtual photon loops cancel out in the ratio (1). While individual loop contributions exhibit IR divergences, cancellation takes place once all terms that contribute to the ratio have been combined. We use a small photon mass $m_{\gamma}$ and electron mass $m_{e}$ to regulate the soft and collinear divergences appearing at intermediate steps, respectively. The real photon radiation contributions require a separate treatment and depend on the kinematic acceptances specific to the experiment. We do not consider them here.

There are also UV divergences, for which we employ dimensional regularization. The UV divergences are eliminated by appropriate renormalization conditions. We employ a renormalization scheme similar to Ref. [15], where the on-shell (OS) scheme is used for the electromagnetic coupling and the $Z$ boson, Higgs boson, and fermion masses. For the weak mixing angle $\sin ^{2} \theta_{W}$, we use the $\overline{\mathrm{MS}}$ renormalization scheme to make contact with descriptions of $\sin ^{2} \theta_{W}$ as a running parameter in the literature. Specifically, we use the $\overline{\mathrm{MS}}$ scheme in the full SM without any decoupled degrees of freedom, which ensures that $\sin ^{2} \theta_{W}(\mu)_{\overline{\mathrm{MS}}}$ is gauge-invariant. By default, the scale choice $\mu=m_{Z}$ is used in the following. Expressions for the OS counterterms can be found in Ref. [21].

To guarantee the cancellation of UV divergences, one must impose the relation $\sin ^{2} \theta_{W}=1-m_{W}^{2} / m_{Z}^{2}$, where 
$m_{W}$ and $m_{Z}$ are the renormalized gauge boson masses in any given renormalization scheme (not necessarily the OS scheme). This implies that one cannot choose an independent renormalization condition for $m_{W}$, but instead the $m_{W}$ counterterm is restricted to

$$
\delta m_{W}^{2}=\left(1-s_{W}^{2}\right) \delta m_{Z, \mathrm{OS}}^{2}-m_{Z}^{2} \delta s_{W, \overline{\mathrm{MS}}}^{2},
$$

where $s_{W}^{2} \equiv \sin ^{2} \theta_{W}(\mu)_{\overline{\mathrm{MS}}}$. The renormalized mass, $m_{W \text {,ren }}$, defined in this fashion differs from the OS mass, $m_{W, O S}$, and an additional finite correction would be needed to relate the two. However, given that $m_{W}$ is never used as an input or output in our calculation, this correction is never explicitly needed in our case.

When performing calculations in dimensional regularization, one has to be careful about the treatment of $\gamma_{5}$. In $d \neq 4$ dimensions, the anticommutation rule $\left\{\gamma^{\mu}, \gamma_{5}\right\}$ is incompatible with the trace identity $\operatorname{tr}\left\{\gamma^{\alpha} \gamma^{\beta} \gamma^{\gamma} \gamma^{\delta} \gamma_{5}\right\}=-4 i \varepsilon^{\alpha \beta \gamma \delta}$. Contributions from such traces arise from vertex diagrams with a triangle subloop [see Fig. 1(e)] and from box diagrams. However, in both of these cases, contributions stemming from $\varepsilon$ tensors are UV finite (after including the subloop counterterms for the box graphs) and thus can be computed in 4 dimensions without ambiguity. (A similar argument holds for a set of useful identities for 4-fermion scattering matrix elements [22,23].) Also, we have confirmed that the triangle diagrams with photons and $\mathrm{Z}$ bosons vanish in the limit of vanishing fermion masses, as required by the anomaly cancellation condition.

Throughout the calculation, we exploit the hierarchy of scales $m_{e}^{2} \ll Q^{2} \ll m_{\text {weak }}^{2}$, where $m_{\text {weak }} \sim m_{W}, m_{Z}, m_{H}, m_{t}$. In practice, this means that the mass of the external electrons is set to zero everywhere except where it is needed to regularize collinear singularities. Furthermore, we perform a large-mass expansion for $m_{\text {weak }}^{2} \gg Q^{2}$, up to order $m_{\text {weak }}^{-2}$, which is the leading order needed for PV effects $[14,15]$. This expansion is based on the method of regions [24-26], and in many cases it leads to products of one-loop integrals and two-loop vacuum integrals, which are analytically known [27-30]. The only exceptions are vertex and box diagrams with a light fermion loop inside a photon or photon-Z propagator [see Fig. 1(b)]. Here, "light fermion" refers to any SM fermion except the top quark.

We evaluate these two-loop integrals using the numerical dispersion integral technique [31] (see also Refs. [32,33]). Since only the transverse part of the subloop self-energy $\Sigma_{\mu \nu}\left(k^{2}\right)$ contributes, we decompose it as

$$
\begin{gathered}
\Sigma_{\mu \nu}=\left[g_{\mu \nu} k^{2}-k_{\mu} k_{\nu}\right] \Pi_{T}\left(k^{2}\right), \\
\Pi_{T}\left(k^{2}\right)=c_{\varepsilon}+\frac{k^{2}}{\pi} \int_{0}^{\infty} d \sigma \frac{\operatorname{Im}\left\{\Pi_{T}(\sigma)\right\}}{\sigma\left(\sigma-k^{2}-i 0\right)} .
\end{gathered}
$$

The contribution of a fermion with mass $m_{f}$ is given by

$$
\begin{gathered}
c_{\varepsilon}=\frac{N_{c} g_{1} g_{2}}{12 \pi}\left(\frac{1}{\varepsilon}+\ln \frac{\mu^{2}}{m_{f}^{2}}\right), \\
\operatorname{Im}\left\{\Pi_{T}(\sigma)\right\}=\frac{N_{c} g_{1} g_{2}}{12 \pi}\left(1+\frac{2 m_{f}^{2}}{\sigma}\right) \\
\times \sqrt{1-\frac{4 m_{f}^{2}}{\sigma}} \Theta\left(\sigma-4 m_{f}^{2}\right),
\end{gathered}
$$

where $1 / \varepsilon=2 /(4-d), \Theta(x)$ is the Heaviside step function, and $N_{c}=1$ (3) for leptons (quarks). The couplings are $g_{1} g_{2}=e^{2} Q_{f}^{2}$ and $g_{1} g_{2}=\left\{\left[e^{2} Q_{f}\left(2 s_{W}^{2} Q_{f}-I_{3 f}\right)\right] /\left[2 s_{W} c_{W}\right]\right\}$ for the photon and photon- $Z$ self-energy, respectively. Inserting these expressions into the outer loop leads to integrals of the form

$$
\begin{aligned}
& \int \frac{d^{d} k}{i \pi^{d / 2}} \frac{N(k)}{\prod_{i}\left[\left(k+p_{i}\right)^{2}-m_{i}^{2}+i 0\right]} \\
& \times\left[c_{\varepsilon}-\frac{1}{\pi} \int \frac{d \sigma}{\sigma} \operatorname{Im}\left\{\Pi_{T}(\sigma)\right\} \frac{k^{2}}{k^{2}-\sigma+i 0}\right] .
\end{aligned}
$$

Here $p_{i}$ are sets of external momenta, as they appear in a given vertex or box diagram, and $N(k)$ accounts for dot products $\left(k^{2}, k \cdot p_{i}\right)$ and $\not k$ in the numerator. The $k$ integral in Eq. (8) is a conventional one-loop integral, which can be performed analytically and reduced to basic scalar one-loop functions using the standard Passarino-Veltman method. The remaining $\sigma$ integral, which is UV finite, is easily evaluated numerically with high precision. It is interesting to note that the $\sigma$ integrals involving $\log m_{\gamma}^{2}$ and $\log m_{e}^{2}$ may be performed analytically so that the cancellation of IR singularities in the full result can be checked algebraically.

These dispersion integrals are not well-defined for light quarks $(f=u, d, s)$ in the inner loop since the dominant contribution to the integral arises from a region where $k^{2} \sim m_{f}^{2}$ and where hadronization effects become important. In fact, the same problem already occurs at the one-loop level for the self-energy contribution to the $\gamma$ - $Z$ self-energy in the $t$ and $u$ channel $[15,34]$ due to the fact that $Q^{2}<\Lambda_{\mathrm{QCD}}^{2}$.

The nonperturbative hadronic corrections can be evaluated only approximately. One option is to use effective quark masses such as the threshold masses derived in Refs. [8,9]. An alternative possibility is to determine the dispersion integrals by directly inserting the required spectral functions derived from data that are provided, e.g., in Ref. [35]. We opted not to follow this method since no uncertainty associated with the required flavor symmetry hypothesis is reported [36]. Therefore, in our calculation, we use the threshold quark masses in all places where mass-dependent terms remain after expanding in large $m_{\text {weak }}^{2}$. However, for consistency, we exclude twoloop self-energy diagrams involving only quark and photon propagators in the loops, such as in Fig. 1(a) with a photon inside the loop, since QED effects are already subsumed in 
the nonperturbative hadron dynamics. In addition, following Ref. [15], we also set $Q^{2} \rightarrow 0$ in the $t$-channel and $u$ channel self-energies since the differences $\Pi_{T}^{\gamma \gamma}(t)-\Pi_{T}^{\gamma \gamma}(0)$ and $\Pi_{T}^{\gamma Z}(t)-\Pi_{T}^{\gamma Z}(0)$ are estimated to be negligibly small [15] (similar for $t$ replaced by $u$ ). We leave a more detailed study of hadronic effects for future work.

As shown in Eq. (2), $A_{L R}$ is commonly normalized in terms of the Fermi constant $G_{\mu}$, which is related to SM parameters according to

$$
\frac{G_{\mu}}{\sqrt{2}}=\frac{\pi \alpha}{2 s_{W}^{2} c_{W}^{2} m_{Z}^{2}}(1+\Delta r)
$$

where $\Delta r$ includes radiative corrections. The required twoloop contributions to $\Delta r$ with one or two closed fermion loops have been taken from Refs. [21,37] (see also Ref. [38]).

The calculation has been carried out with extensive use of computer algebra tools. Diagrams and amplitudes were generated with FEYNARTS [39]. For the Lorentz and Dirac algebra, we employed PACKAGE-X [40] and cross-checked against private code written in MATHEMATICA. The largemass expansion was implemented in-house in two independently developed MATHEMATICA programs. Two-loop integrals with nontrivial numerator structures have been reduced to simple scalar integrals using FIRE 5 [41] and using private code based on Ref. [42,43]. For basic oneloop integrals and two-loop vacuum integrals, analytical formulas are available [27-30]. We have numerically checked our implementation of the one-loop formulas against the COLLIER library [44]. The numerical dispersion integrals for two-loop vertex and box integrals have been implemented in C and MATHEMATICA.

Each building block of the final result has been computed in two independent setups within our collaboration and crosschecked against each other. We have confirmed cancellation of UV and IR divergences in the full result by verifying that the coefficients of the $1 / \varepsilon, \log m_{e}^{2}$, and $\log m_{\gamma}^{2}$ terms vanish algebraically. Furthermore, as an intermediate step, we have reproduced the one-loop result of Ref. [15] and found exact agreement with the analytical formulas given there.

Results.-To evaluate the numerical impact of the closed fermion loop NNLO corrections to $A_{L R}$, we used the following input parameters:

$$
\begin{aligned}
m_{Z} & =91.1876 \mathrm{GeV}, \quad s_{W}^{2}=0.2314, \\
m_{H} & =125.1 \mathrm{GeV}, \quad m_{t}=173.0 \mathrm{GeV}, \\
m_{\tau} & =1.777 \mathrm{GeV}, \quad m_{b}=3.99 \mathrm{GeV}, \\
m_{\mu} & =105.7 \mathrm{MeV}, \quad m_{c}=1.185 \mathrm{GeV}, \\
m_{e} & =0.511 \mathrm{MeV}, \quad m_{s}=0.342_{-0.053}^{+0.048} \mathrm{GeV}, \\
m_{u, d} & =0.246_{-0.057}^{+0.054} \mathrm{GeV}, \\
\Delta \alpha & =0.02761_{\mathrm{had}}+0.0314976_{\mathrm{lep}},
\end{aligned}
$$

at the representative kinematic point

$$
\sqrt{s}=11 \mathrm{MeV}, \quad y=0.4 \text {. }
$$

Here $\Delta \alpha$ accounts for the renormalization group running of the fine structure constant between scales $\mu=0$ and $\mu=m_{Z}$ and enters our calculation through the OS charge renormalization. The first number reflects the hadronic contribution to $\Delta \alpha$, which is obtained from $e^{+} e^{-} \rightarrow$ hadrons data (see Refs. [35,45,46] for recent evaluations), while the second number is the perturbatively calculable leptonic contribution [47].

As explained above, the light fermion masses $m_{f}, f \neq t$ enter in loop integrals with a fermionic photon or $\gamma-Z$ selfenergy subloop. The values for the light quark masses are taken from Ref. [9]. There is a strong anticorrelation between the reported uncertainties of $m_{s}$ and $m_{u, d}$. We will assume them to be $100 \%$ anticorrelated for the results that we present below.

With these inputs, we obtain numerical results for the asymmetry (2) as shown in Table I. The first row corresponds to the tree level contribution, and the remaining rows $\Delta Q_{W\left(L, n_{f}\right)}^{e}$ are the radiative corrections with $L$ loops and $n_{f}$ closed fermion loops. No resummation of logarithms has been carried out. In particular, the electroweak logarithms, which conventionally define the running $\sin ^{2} \theta_{W}$, are left explicitly in the one- and two-loop results. The last two rows $\Delta Q_{W(2,2)}^{e}$ and $\Delta Q_{W(2,1)}^{e}$ are obtained using our newly computed NNLO corrections to the asymmetry. The error intervals reflect the hadronic uncertainties due to the threshold quark masses in Eq. (10).

The precision goal for the MOLLER experiment corresponds to a measurement of the weak charge with an uncertainty of $\delta_{\exp } Q_{W}^{e}=1.1 \times 10^{-3}$. The NNLO corrections with closed fermion loops add up to

$$
\Delta Q_{W(2,2)}^{e}+\Delta Q_{W(2,1)}^{e}=1.00_{-0.008}^{+0.012} \times 10^{-3},
$$

which is comparable to the experimental target, thus highlighting the importance of accounting for the NNLO

TABLE I. Numerical estimates of the calculated contributions to the polarized Møller scattering asymmetry defined in Eq. (2) through NNLO using input values in Eqs. (10) and (11). Subscripted indices on $\Delta Q_{W\left(L, n_{f}\right)}^{e}$ refer to the loop order $L$ and the number of closed loops $n_{f}$.

\begin{tabular}{ll}
\hline \hline Quantity & Contribution $\left(\times 10^{-3}\right)$ \\
\hline $1-4 \sin ^{2} \theta_{W}$ & +74.4 \\
$\Delta Q_{W(1,1)}^{e}$ & -29.0 \\
$\Delta Q_{W(1,0)}^{e}$ & +3.1 \\
$\Delta Q_{W(2,2)}^{e}$ & $-0.18_{-0.0040}^{+0.0024}$ \\
$\Delta Q_{W(2,1)}^{e}$ & $+1.18_{-0.010}^{+0.015}$ \\
$\Delta Q_{W(2,0)}^{e}$ & \pm 0.13 (estimate) \\
\hline \hline
\end{tabular}


corrections. On the other hand, anti-correlation between the hadronic uncertainties in the individual contributions with one and two closed fermion loops leads to a reduced overall hadronic uncertainty.

The resulting hadronic uncertainty from quark loops is negligible compared to the experimental target precision. It is likely that the our estimate based on quark mass errors overestimates this uncertainty since we cannot account for correlations between the quark masses and the $K$ factors in Ref. [9]. (In fact, when estimating the leading hadronic effects by plugging these quark masses into the NLO correction, one finds an uncertainty that is a few factors larger than the detailed renormalization-group evaluation in Ref. [9].) A more detailed analysis of hadronic effects will be given in a future publication.

The correction $\Delta Q_{W}^{e}$ depends very mildly on $y$ (i.e., on the scattering angle). Varying $y$ in the experimentally relevant range $(0.25,0.75)$ [6], we find that $\Delta Q_{W}^{e}$ changes by $0.04 \times 10^{-3}$ for the NLO corrections and by $0.01 \times 10^{-3}$ for the NNLO corrections, both of which are negligible.

Finally, we attempt to estimate the size of the currently missing NNLO corrections without closed fermion loops $\Delta Q_{W(2,0)}^{e}$ (called "bosonic" corrections in the following). For this purpose, we begin by comparing the relative size of the fermion loop and bosonic correction at NLO. From Table I, these are $\Delta Q_{W(1,1)}^{e}=-0.0290$ and $\Delta Q_{W(1,0)}^{e}=+0.0031$, respectively. Assuming a similar ratio between the corrections with one closed fermion loop and the bosonic corrections at NNLO, we obtain an estimate of $0.13 \times 10^{-3}$ for the size of the latter. This would be safely below the experimental target precision.

Conclusions. - To correctly interpret the proposed 2.4\% measurement of the PV asymmetry $A_{L R}$ from the MOLLER experiment at the Jefferson Lab in terms of BSM physics, we calculate the NNLO SM contributions to $A_{L R}$ using large-mass expansion and numerical integration of subloop dispersion relation. We summarize our results in Table I. We find that the corrections to $\Delta Q_{W}^{e}$ from diagrams with closed fermion loops are comparable to the experimental target precision. The dependence of $\Delta Q_{W}^{e}$ on the scattering angle is very mild in the experimentally relevant range and can be ignored for most practical purposes. Finally, we also consider the impact of the remaining bosonic NNLO corrections and estimate them to be negligible compared to the MOLLER precision goal. However, it is desirable to confirm this with an explicit calculation in the future.

The authors would like to thank V. A. Smirnov and J. Erler for useful private communications. Y. D. is grateful to K. Kumar for his financial support at Fermilab and to PITT-PACC at the University of Pittsburgh for their hospitality during part of this work. A.F. has been supported in part by the National Science Foundation under Grant No. PHY-1820760. Y.D., H. H.P., and
M. J. R.-M. were supported in part under U.S. Department of Energy Contract No. DE-SC0011095. H.H.P. was additionally supported in part by U.S. Department of Energy Award No. DE-SC0010107.

* Corresponding author. yongdu@umass.edu Corresponding author. afreitas@pitt.edu Corresponding author. hpatel6@ucsc.edu

${ }^{\S}$ Corresponding author. mjrm@physics.umass.edu

[1] S. L. Glashow, Partial symmetries of weak interactions, Nucl. Phys. 22, 579 (1961).

[2] A. Salam, Weak and electromagnetic interactions, Conf. Proc. C 680519, 367 (1968).

[3] S. Weinberg, A Model of Leptons, Phys. Rev. Lett. 19, 1264 (1967).

[4] P. L. Anthony et al. (SLAC E158 Collaboration), Precision Measurement of the Weak Mixing Angle in Moller Scattering, Phys. Rev. Lett. 95, 081601 (2005).

[5] J. Mammei et al.(MOLLER Collaboration), The MOLLER Experiment, Nuovo Cimento C 035N04, 203 (2012).

[6] J. Benesch et al. (MOLLER Collaboration), The MOLLER Experiment: An ultra-precise measurement of the weak mixing angle using Møller scattering, http://arxiv.org/abs/ 1411.4088.

[7] A. Czarnecki and W. J. Marciano, Parity violating asymmetries at future lepton colliders, Int. J. Mod. Phys. A 13, 2235 (1998).

[8] J. Erler and M. J. Ramsey-Musolf, The weak mixing angle at low energies, Phys. Rev. D 72, 073003 (2005).

[9] J. Erler and R. Ferro-Hernández, Weak mixing angle in the Thomson limit, J. High Energy Phys. 03 (2018) 196.

[10] P. S. Bhupal Dev, M. J. Ramsey-Musolf, and Y. Zhang, Doubly-charged scalars in the type-II Seesaw mechanism: Fundamental symmetry tests and high-energy searches, Phys. Rev. D 98, 055013 (2018).

[11] H. Davoudiasl, H.-S. Lee, and W. J. Marciano, Muon Anomaly and Dark Parity Violation, Phys. Rev. Lett. 109, 031802 (2012).

[12] H. Davoudiasl, H.-S. Lee, and W. J. Marciano, Muon $g$ ?2, rare kaon decays, and parity violation from dark bosons, Phys. Rev. D 89, 095006 (2014).

[13] H. Davoudiasl, H.-S. Lee, and W. J. Marciano, Low $Q^{2}$ weak mixing angle measurements and rare Higgs decays, Phys. Rev. D 92, 055005 (2015).

[14] E. Derman and W. J. Marciano, Parity violating asymmetries in polarized electron scattering, Ann. Phys. (N.Y.) 121, 147 (1979).

[15] A. Czarnecki and W. J. Marciano, Electroweak radiative corrections to polarized Moller scattering asymmetries, Phys. Rev. D 53, 1066 (1996).

[16] A. Aleksejevs, S. Barkanova, Y. Kolomensky, E. Kuraev, and V. Zykunov, Quadratic electroweak corrections for polarized Moller scattering, Phys. Rev. D 85, 013007 (2012). 
[17] A. G. Aleksejevs, S. G. Barkanova, Y. M. Bystritskiy, A. N. Ilyichev, E. A. Kuraev, and V. A. Zykunov, Double-box contributions to Moeller scattering in the standard model, Eur. Phys. J. C 72, 2249 (2012).

[18] A. G. Aleksejevs, S. G. Barkanova, Yu. M. Bystritskiy, E. A. Kuraev, and V. A. Zykunov, NNLO Electroweak corrections for polarized Møller scattering: One-loop insertions to boxes, Phys. Part. Nucl. Lett. 12, 645 (2015).

[19] A. G. Aleksejevs, S. G. Barkanova, Yu. M. Bystritskiy, E. A. Kuraev, and V. A. Zykunov, Two-loop electroweak vertex corrections for polarized Møller scattering, Phys. Part. Nucl. Lett. 13, 310 (2016).

[20] R.-D. Bucoveanu and H. Spiesberger, QED radiative corrections for polarized lepton-proton scattering, Proc. Sci., SPIN2018 (2019) 115 [arXiv:1903.12229].

[21] A. Freitas, W. Hollik, W. Walter, and G. Weiglein, Electroweak two loop corrections to the $M_{W}-M_{Z}$ mass correlation in the standard model, Nucl. Phys. B632, 189 (2002); Erratum, Nucl. Phys. B666, 305 (2003).

[22] A. Sirlin, A class of useful identities involving correlated direct products of $\gamma$ matrices, Nucl. Phys. B192, 93 (1981).

[23] A. Denner, S. Dittmaier, M. Roth, and L. H. Wieders, Electroweak corrections to charged-current $\mathrm{e}+\mathrm{e}-\longrightarrow$ fermion processes: Technical details and further results, Nucl. Phys. B724, 247 (2005); Erratum, Nucl. Phys. B854, 504 (2012).

[24] M. Beneke and V. A. Smirnov, Asymptotic expansion of Feynman integrals near threshold, Nucl. Phys. B522, 321 (1998).

[25] V. A. Smirnov, Problems of the strategy of regions, Phys. Lett. B 465, 226 (1999).

[26] V. A. Smirnov, Applied asymptotic expansions in momenta and masses, Springer Tracts Mod. Phys. 177, 1 (2002).

[27] G. 't Hooft and M. J. G. Veltman, Scalar one loop integrals, Nucl. Phys. B153, 365 (1979).

[28] W. Beenakker and A. Denner, Infrared divergent scalar box integrals with applications in the electroweak standard model, Nucl. Phys. B338, 349 (1990).

[29] C. Ford, I. Jack, and D. R. T. Jones, The Standard Model effective potential at two loops, Nucl. Phys. B387, 373 (1992); Erratum, Nucl. Phys. B504, 551 (1997).

[30] A. I. Davydychev and J. B. Tausk, Two loop selfenergy diagrams with different masses and the momentum expansion, Nucl. Phys. B397, 123 (1993).

[31] S. Bauberger, F. A. Berends, M. Bohm, and M. Buza, Analytical and numerical methods for massive two loop selfenergy diagrams, Nucl. Phys. B434, 383 (1995).

[32] M. Awramik, M. Czakon, and A. Freitas, Electroweak twoloop corrections to the effective weak mixing angle, J. High Energy Phys. 11 (2006) 048.
[33] A. Aleksejevs, Dispersion approach in two-loop calculations, Phys. Rev. D 98, 036021 (2018).

[34] W. J. Marciano, Spin and precision electroweak physics, in Spin Structure in High-Energy Processes: Proceedings, 21st SLAC Summer Institute on Particle Physics, 1993, Stanford, CA, edited by L. DePorcel and C. Dunwoodie (1995), http://www.slac.stanford.edu/spires/find/books/ www?cl=QCD161:S76:1993.

[35] F. Jegerlehner, Variations on photon vacuum polarization, EPJ Web Conf. 218, 01003 (2019).s

[36] However, one can verify that the running behavior of the weak coupling derived in Ref. [35] can be almost exactly reproduced by a suitable choice of effective quark masses. The difference between this choice of quark masses and those of Ref [9] leads to a shift of the two-loop corrections to $A_{L R}$ that is 1 order of magnitude below the expected level of precision of MOLLER.

[37] A. Freitas, W. Hollik, W. Walter, and G. Weiglein, Complete fermionic two loop results for the $\mathrm{M}(\mathrm{W})-\mathrm{M}(\mathrm{Z})$ interdependence, Phys. Lett. B 495, 338 (2000); Erratum, Phys. Lett. B 570, 265 (2003).

[38] M. Awramik and M. Czakon, Complete two loop electroweak contributions to the muon lifetime in the standard model, Phys. Lett. B 568, 48 (2003).

[39] T. Hahn, Generating Feynman diagrams and amplitudes with Feynarts 3, Comput. Phys. Commun. 140, 418 (2001).

[40] H. H. Patel, Package-X: A Mathematica package for the analytic calculation of one-loop integrals, Comput. Phys. Commun. 197, 276 (2015).

[41] A. V. Smirnov, FIRE5: A C ++ implementation of Feynman integral reduction, Comput. Phys. Commun. 189, 182 (2015).

[42] G. Weiglein, R. Scharf, and M. Bohm, Reduction of general two loop selfenergies to standard scalar integrals, Nucl. Phys. B416, 606 (1994).

[43] A. I. Davydychev and J. B. Tausk, Tensor reduction of two loop vacuum diagrams and projectors for expanding three point functions, Nucl. Phys. B465, 507 (1996).

[44] A. Denner, S. Dittmaier, and L. Hofer, Collier: A fortranbased complex one-loop library in extended regularizations, Comput. Phys. Commun. 212, 220 (2017).

[45] M. Davier, A. Hoecker, B. Malaescu, and Z. Zhang, A new evaluation of the hadronic vacuum polarisation contributions to the muon anomalous magnetic moment and to $\boldsymbol{\alpha}\left(\mathbf{m}_{\mathbf{Z}}^{2}\right)$, Eur. Phys. J. C 80, 241 (2020).

[46] A. Keshavarzi, D. Nomura, and T. Teubner, The $g-2$ of charged leptons, $\alpha\left(M_{Z}^{2}\right)$ and the hyperfine splitting of muonium, Phys. Rev. D 101, 014029 (2020).

[47] M. Steinhauser, Leptonic contribution to the effective electromagnetic coupling constant up to three loops, Phys. Lett. B 429, 158 (1998). 